\title{
Note on Varying Speed of Light Cosmologies
}

\author{
George F R Ellis. \\ Mathematics Department and Applied Mathematics, \\ University of Cape Town, South Africa.
}

March 5, 2018

\begin{abstract}
The various requirements on a consistent varying speed of light ('VSL') theory are surveyed, giving a short check-list of issues that should be satisfactorily handled by such theories.
\end{abstract}

\section{$1 \quad$ Varying Speed of Light ('VSL') Cosmologies}

Many papers have been published on varying speed of light ('varying $c$ ') theories, with claims inter alia that they can solve some of the problems that inflationary cosmologies seek to solve. However many of those papers are problematic, and the increasing literature in the area does not fully take cognisance of some foundational issues for any VSL theory.

This note will not comment on any specific such papers in detail, although it will use a few as examples of what is discussed. Rather it will aim to provide the reader with a toolkit enabling a critique of any VSL paper, checking if it meets some basic criteria that can be claimed to be fundamental for an adequate discussion of VSL proposals. A key point will be distinguishing theories where it is the speed of light $v_{\text {photon }}$ - the speed at which photons travel - that is supposed to vary, and those where it is a universal causally limiting speed $v_{\text {lim }}$ that is supposed to vary. While these are the same speed $c$ in standard relativity theory, they can and indeed probably will differ in any genuine VSL theory.

One might ask why it is necessary to place an article containing material that seems rather elementary in a research journal. The reason is that VSL papers that ignore these fundamentals are continually being put on the archive and even being published in reputable journals. This paper will hopefully help future work in the area avoid common pitfalls in formulating such theories.

\section{Fundamentals: the speed of light and measurement}

First, note that just because a quantity is called 'the speed of light' and labeled 'c' does not necessarily mean it is the speed of light in physical terms. What determines that some quantity is in fact the physical speed of light $v_{\text {photon }}$ ? In standard relativity theory [5] this is related on the one had to the metric tensor, which determines time and spatial measurements as well as the geometry of null geodesics, and on the other hand to Maxwell's equations, which determine the paths of light rays in space time. These are linked because the characteristics of wavelike solutions of Maxwell's equations are null geodesics, as determined by the metric tensor. Thus in standard General Relativity ('GR'), it is a fundamental interpretational principle that light rays - the paths of photons and other massless particles in spacetime - are solutions of the equation

$$
d s^{2} \equiv g_{\mu \nu}\left(x^{k}\right) d x^{\mu} d x^{\nu}=0,
$$

which therefore determines the speed of light $v_{\text {photon }}$.

Second, meaningful variations in the constants of nature refer only to dimensionless constants, because only dimensionless quantities have an invariant meaning when units of measurement are changed. The quantity ' $c$ ' is not dimensionless; consequently, one can change the apparent speed of light to any desired value by changing the coordinates or the units used. This does not mean the physical speed of light has changed. Indeed one can always set the speed of light to unity by appropriate choice of units: simply rescale either spatial distances or time units, as one prefers, and one can set any physically measured speed of light to unity. Thus for example if the metric of Minkowski spacetime is initially given as

$$
d s^{2}=-d t^{2}+d r^{2} / c^{2}, d r^{2} \equiv d x^{2}+d y^{2}+d z^{2}
$$


then ' $c$ ' is indeed the speed of light $v_{\text {photon }}$, as (1) implies

$$
d s^{2}=0 \Rightarrow\left(v_{\text {photon }}\right)^{2}=d r^{2} / d t^{2}=c^{2} .
$$

Now one can rescale: $X=x / c, Y=y / c, Z=z / c$, to get

$$
d s^{2}=-d t^{2}+d R^{2}, d R^{2} \equiv d X^{2}+d Y^{2}+d Z^{2},
$$

and the speed of light has been set to unity:

$$
d s^{2}=0 \Rightarrow\left(v_{\text {photon }}\right)^{2}=d R^{2} / d t^{2}=1,
$$

without any loss of generality of the physics involved. By similar transformations we can make the speed of light anything we want; this is not a puzzle, as of course this is just the coordinate speed of light.

The key issue relating this to physics is how one measures spatial distances and times, for it is only when we have distance and time units set up that we can start to measure the speed of light. One can claim [6 that on macroscopic scales - from meters to solar system scale - the only practical way of determining distance is via radar; other astronomical distance scales are derived from this one 11 One assumes a good clock can be constructed, and then uses the timing of reflected electromagnetic radiation to determine the distance. But then the (physical) speed of light of necessity has to be unity, precisely because all electromagnetic radiation travels at the speed of light, and distances are being determined by use of such radiation. This is reflected in the natural units used for such distance measurements: light seconds and light years. When such units are used, the speed of light is unity by definition - not by definition of how fast light moves, but by the definition used for spatial distances 2 From a practical point of view, the speed of light has been fixed by the BIPM (Bureau International des Poids et Mesures), and we derive the meter from it. Then equation (11) does not determine the speed of light - it defines it.

Implication 1: In order to be viable, any VSL theory involving a variable speed of photon travel must of necessity be based on some other method of measuring spatial distances than radar. So the question for any specific proposed VSL theory is, What viable alternative proposal for distance measurement is made:3

\section{The speed of light and the metric}

In terms of the metric tensor components, in standard relativity theory we are allowed to make basis transformations that are not Lorentz transformations; and they can make the metric tensor components anything we want! This is essentially what is being done in cosmology when the Robertson-Walker metric tensor

$$
d s^{2}=-c_{0}^{2} d t^{2}+a^{2}(t) d \sigma^{2}, d \sigma^{2} \equiv d r^{2}+f^{2}(r)\left(d \theta^{2}+\sin ^{2} \theta d \phi^{2}\right)
$$

is assumed to hold initially, and then postulated to change to

$$
d s^{2}=-c_{1}^{2} d t^{2}+a^{2}(t) d \sigma^{2}
$$

at some critical time $t_{*}$ in the early universe, where $c_{i}$ are constants 4 Then (6) implies on radial null geodesics

$$
d s^{2}=0 \Rightarrow v_{\text {photon }}=\left(1 / c_{0}\right) a(t) d r / d t,
$$

while (7) implies on radial null geodesics

$$
d s^{2}=0 \Rightarrow v_{\text {photon }}=\left(1 / c_{1}\right) a(t) d r / d t,
$$

giving an apparent change in the speed of light by a factor $c_{0} / c_{1}$. However in fact the units of measurement have been changed (leading to a consequent change in the metric tensor components), rather than the physical speed of light altering. Indeed one can transform (77) to (6) by the change of coordinates $t \rightarrow\left(c_{0} / c_{1}\right) t$. So according to the principle of general covariance, they are just the same spacetime in different coordinates (this feature being confused by using the same label ' $t$ ' for what are in fact two different time coordinates used at earlier and later times). The two metrics do not represent different physical speeds of light.

Is there some preferred time coordinate that can break this degeneracy in the coordinate speed of light? Yes indeed; proper time $\tau$ is by definition the time measured along a (timelike) world line by a perfect clock. In both standard special relativity ('SR') and GR, it is give by the line integral

$$
\tau=\int d \tau, d \tau \equiv \sqrt{-d s^{2}}=\sqrt{-g_{\mu \nu}\left(x^{k}\right) d x^{\mu} d x^{\nu}} .
$$

\footnotetext{
${ }^{1}$ Parallax distance measurements for example rely on knowing the physical size of the base used to determine the parallax; and that has to be determined by some method such as radar.

${ }^{2}$ Indeed Synge has emphasized that time is the fundamental measurement: all classical physical quantities can be expressed in terms of units of time (11], see particularly Appendix B.)

${ }^{3}$ The majority of VSL papers do not discuss this issue.

${ }^{4} \mathrm{~A}$ 'phase change' is supposed to take place. This is essentially what is proposed in [9], see eqns. (18)-(20).
} 
Applying this to the fundamental world lines with tangent vector $u^{a}=\delta_{0}^{a} / c_{0}$ in (6) reveals that in that metric proper time along these world lines is $\tau=c_{0} t$. Similarly (7) reveals the proper time $\tau$ along these world lines is $\tau=c_{1} t$. The quantity (10) is an invariant, and will be the same whatever coordinate system is used. While we can use any coordinates, some are more convenient than others in that they more directly represent the physics of what is going on; we get these preferred coordinates on choosing the time coordinate $t$ as proper time $\tau$ along the fundamental world lines at all times. Then $c_{0}=1$ and $c_{1}=1$, (6) is identical to (7), and there is no jump in the apparent speed of light. If there is a spacetime where the metric tensor components change at some time $t_{*}$ from the form (66) to (7) with $c_{o} \neq c_{1}$, nothing has changed physically; there has simply been a rescaling in the time coordinate used. This has not affected the physical speed of light.

Similarly spatial distances are determined by the integral of the line element $\sqrt{d s^{2}}$ along spacelike curves. This prescription together with (10) gives a relation between time and spatial distance measurements compatible with the radar definition of distance mentioned in the previous section, and so is a way of codifying the feature that the speed of light is invariant in standard GR.

Implication 2: Any varying speed of light theory based on changes in the metric tensor components must explain how it differs from GR as regards how time and space measurements are related to the metric tensor. What replaces (10) in the proposed theory?

\section{The speed of light, causality, and the Lorentz group}

The speed of light plays a key role in standard physics because it is the limiting speed $v_{l i m}$ for local relative motion, as indicated by the standard relativistic laws for velocity transformations derived from the Lorentz group. This is indicated by the way it is a universal speed, invariant under velocity addition:

$$
\forall v: v+v_{l i m} \rightarrow v_{l i m} .
$$

It is consequently a speed that cannot be exceeded by any physical object or information-carrying signal, and this is related for example to the increase of inertial mass with relative motion, diverging as this speed is approached. Thus $v_{\text {lim }}$ determines local causality. This basically occurs because physics is Lorentz invariant, and this limiting speed is uniquely characterized by the fact that it is Lorentz invariant; indeed this is what underlay the initial derivation of the Lorentz group.

The link to the metric tensor is that Lorentz transformations are precisely those transformations that leave the metric tensor components invariant. A key role is played by the relation between the contravariant and covariant metric tensor components:

$$
g_{a b} g^{b c}=\delta_{a}^{c}
$$

which relates the speed of light in these two sets of components: using Synge's convention [1] that time measurements will be fundamental, at any point the metric components can be written as

$$
g_{a b}=\operatorname{diag}\left\{-1,1 / c^{2}, 1 / c^{2}, 1 / c^{2}\right\} \Rightarrow g^{a b}=\operatorname{diag}\left\{-1, c^{2}, c^{2}, c^{2}\right\} .
$$

Lorentz transformations preserve both these canonical metric tensor forms. The limiting causal speed $v_{l i m}$ is just the 'speed of light' constant $c$ that occurs in both these metric forms and is left invariant by the Lorentz group. It consequently occurs in the Lorentz factor

$$
\gamma(v)=1 / \sqrt{1-v^{2} / c^{2}}
$$

characterizing the kinematic effects of length contraction and time dilation as well as the dynamic effect of increasing inertial mass; indeed the standard time dilation formula follows immediately from the conjunction of (10) with (13) (see [6]), and the dynamical limits on speed follow from the relativistic increase in mass: $m=m_{0} \gamma(v)$. But this limiting speed can always be set to unity, globally in SR as explained above (see the transformation from (2) to (44) above), and locally at any point in GR (in essence, because of the principle of equivalence together with the special relativity result). Natural units for spatial distances and time, associated with normalized coordinates and associated orthonormal bases of vectors, indeed give this normalized form (4) at any point:

$$
g_{a b}=\operatorname{diag}\{-1,1,1,1\}, g^{a b}=\operatorname{diag}\{-1,1,1,1\},
$$

showing explicitly that the limiting speed $v_{l i m}$ (which is the quantity ' $c$ ' in (13) and (14)) is unity; as is required for consistency, $\gamma(v)=1 / \sqrt{1-v^{2}}$.

In the cosmological context, we can if we wish change the time variable $t$ in the metric (6) to $\eta=\int d t / a(t)$ to obtain the conformally flat metric form

$$
d s^{2}=a^{2}(t)\left\{-d \eta^{2}+d \sigma^{2}\right\} .
$$


By (1), radial light rays plotted in terms of the coordinates $(\eta, r)$ will always be at $\pm 45^{\circ}$; thus the limiting speed $v_{\text {lim }}$ is again found to be unity. These are the coordinates used in the standard Penrose conformal diagrams of cosmology, used to make clear causal relations in these spacetimes [6]. Changing time or space coordinates does not affect these causal relations, which can always be represented by such diagrams with $c=15$

In SR and GR, the equations for all fundamental physical fields are written in Lorentz invariant form. The consequent use of the metric tensor $\sqrt{6}$ in the action or in resultant field equations such as the Klein-Gordon equation implicitly introduces the speed of light $c$, ensuring that causality works out correctly. The wavelike characteristics for massless physical fields are then light rays, because the wave operator

$$
\nabla^{2} f=g^{a b} f_{; a b}
$$

relates these characteristics to the metric tensor, and hence to the value of $c$ occurring in that tensor. Discontinuities in these fields and waves will travel at the speed of light as specified by the metric tensor components (13), and their causal properties will be represented correctly by the Penrose conformal diagrams mentioned above.

Implication 3: Any varying speed of light theory involving a change in the limiting speed will not be Lorentz invariant; the way Lorentz invariance is broken must be made explicit 7 If it involves an alteration of the Lorentz group, it must make clear how the limiting dynamical speed is determined.

\section{$5 \quad$ The speed of light and Maxwell's equations}

Of particular concern in relation to the speed of light is the physics that in fact determines the speed of light. Any adequate varying speed of light theory cannot just postulate ad hoc changes to the speed of light $v_{\text {photon }}$, perhaps assuming it can undergo a 'phase transition' for example, without some physical reason for such a change; doing so is not proposing a coherent physical theory. The physics supposed to underlie the variation of $v_{\text {photon }}$ should be made explicit.

At a minimum one can (in the spirit of present day physics) assume $c$ is a dynamical variable and propose some effective field equations to govern its variation. Any such equations must eventually be derivable either from Maxwell's equations or from whatever other equations will be taken as underlying electromagnetic theory. One can perhaps in an investigative spirit leave that problem for the moment and just assume some effective equations to govern its time and space variation. But that can only be a temporary expedient: eventually if it is indeed the speed of light $v_{\text {photon }}$ we are talking about, it must be related to the equations of electromagnetism 8

In standard relativity theory, the metric tensor is used to raise and lower indices on the Maxwell field tensor $F_{a b}$, and hence relates the two sets of Maxwell's equations:

$$
F_{[a b ; c]}=0, F_{; b}^{a b}=0, F^{a b} \equiv g^{a c} g^{b d} F_{b d}
$$

thus by (13) inserting $c$ in the derivative terms in these equations 9 The divergence relation $F_{; a b}^{a b}=0$ then leads to the standard wave equation for electromagnetism, with $c$, deriving from the metric tensor form (13), being the physical speed of light $v_{\text {photon }}$, and so corresponding to a massless photon. As pointed out above, $c$ can be normalised to unity in the metric form without loss of generality, and then the actual speed of light is a constant rather than a variable quantity.

One can avoid this conclusion in various ways: for example utilising a bimetric theory with one metric determining space and time measurements and another being used in Maxwell's equations 10 invoking a nonminimal coupling between electromagnetism and gravity 11 or proposing a theory of massive photons such as given for example by the Proca action [10. The first key point is that some proposal needs to be made in this regard: when one talks about the speed of light varying, this only gains physical meaning when related to Maxwell's equations or its proposed generalisations, for they are the equations that determine the actual speed of light. The second key point is that just because there is a universal speed $v_{l i m}$ does not prove there is a particle that moves at that speed. On the standard theory, massless particles move at that speed and massive particles don't; but by itself, that result does not imply that massless particles exist. Their existence is a further assumption of the

\footnotetext{
${ }^{5}$ Hence Figure 2 of [1] shows some light cones as flatter than $45^{0}$ because unusual coordinates have been chosen. Choice of conformal coordinates would bring this diagram to standard form with all light rays at $\pm 45^{\circ}$, and hence no variation of the speed of light. The same issue arises with Figure 2 of $[8]$.

${ }^{6}$ For example in raising and lowering indices.

${ }^{7}$ Such a proposal is made in [1] for example,and see [8] for discussion.

${ }^{8}$ See 7 for a clear discussion disentangling the link between SR and electromagnetism.

${ }^{9}$ It may also occur in the relation between the tensor $F_{a b}$ and the electric and magnetic fields $E, B$ as well as the charge $e$ and current $j$. However these occurrences of $c$ are dependent on the units used for $E, B, e$ and $j$, and can be absorbed in those quantities without loss of generality.

${ }^{10}$ For a viable implementation of this idea, see 3 .

${ }^{11}$ For a viable implementation of this idea, see [12.
} 
standard theory, related to the wavelike equations satisfied by the Maxwell electromagnetic field. One can consider theories with massive photons.

Implication 4: Any varying speed of light theory involving a change in the speed of photon travel must eventually propose some other equations than standard Maxwell's equations to govern electromagnetism, and show how this leads to a varying physical speed of light associated with a wavelike solution to these equations.

\section{The speed of light and dynamical equations}

Any proposed variation of the speed of light has major consequences for almost all physics, as it enters many physics equations in various ways, particularly because of the Lorentz invariance built into fundamental physics (see 4 for discussion). One needs to take cognisance of the effects of this variation on the rest of physics.

What is sometimes done is to just allow the constant $c$ to become a variable in one or more physical equations, and then proclaim this as a variable speed of light theory. Three points are relevant here. First, if a combination of 'constants' varies in a dynamical equation, it might be any of them that is responsible. It is only specifically the speed of light that varies if it is related to the physical speed of light as discussed in the previous sections. For instanc $\sqrt{12}$ if one allows the quantity $c$ to become a variable in the equation of state $p=w \rho c^{2}$, it is the combination $\hat{w}=w c^{2}$ that is the physically effective quantity that is made to vary. Indeed one can absorb $c^{2}$ into the definition of the equation of state variable $w$, as this just amounts to a choice of physical units for $p$. Then the normalized equation of state is just $p=\hat{w} \rho$, and what appeared to be a variable speed of light theory can be regarded as just introduction of a time-dependent equation of state. As another example [2, if in

$$
G_{a b}=\kappa T_{a b}+\Lambda g_{a b},
$$

where

$$
\kappa \equiv \frac{8 \pi G}{c^{4}}
$$

one allows the speed of light $c$ to become a variable, then $\kappa$ becomes a variable and this changes the solutions to these gravitational equations. Equivalently, one can propose such a variation in the action

$$
S=\int \sqrt{-g} d x^{4}\left(\frac{R+2 \Lambda}{2 \kappa}+L_{m}\right)
$$

and work out the dynamic consequences. But the point then is that the physical effects contemplated here occur only because $\kappa$ is now varying; and a varying $\kappa$ does not necessarily mean $c$ varies. Indeed if the supposed variation in $\kappa$ is not linked to the other effects mentioned in the previous sections of this note, then $c$ is not varying - because it is those effects that identify the varying quantity to be the speed of light. Rather what one has in fact done is propose a varying $G$ theory - and of course there is a large literature on that topic. If it is to be specifically $c$ that is varying, you must vary it in all places where it occurs in the physical equations, which is many places; but where it occurs in the equations depends on the units used, so it is not obvious how to do this coherently and uniquely. For example in the above equations we can define $\tilde{G} \equiv G / c^{4}$, which amounts to a specific choice of units to be used for $G$; then

$$
\kappa \equiv 8 \pi \tilde{G}
$$

and $c$ does not appear in equations (19), (21), (22). Which form of the equations to use is a free choice; the result of allowing $c$ to vary is quite different depending on the form chosen 13

Secondly, when a quantity is changed from a constant to a function, obtaining the correct dynamic equations requires doing the variation with that quantity treated as a variable ab initio. One gets the wrong result by first doing the variation and then changing the constant to a variable. For example, setting $c^{4}=\psi(x)$ in (20) and adding a term $L_{\psi}$ into the action (21) results in a set of gravitational equations that are not the same as those obtained by first performing the variation and then setting $c^{4}=\psi(x) 14$ This is because the term involving $R$ in (21) is multiplied by $1 / \kappa$, which is then no longer constant. The first method is the one that gives the correct result. Simply setting $c$ to a variable in the dynamic equations after the variation has been performed leads to problems with consistency of the resulting equations, see [3, 4, for discussion.

Finally any such proposed change of $c$ in various physical equations needs some motivation in physical terms: what fundamental physical cause has changed the quantity $c$ ? This has been commented on in the previous section. We just note here that any variable which varies is either a combination of basic fields, or is itself a field in the sense that it has a kinetic term. If one lets a quantity vary in spacetime without giving it a kinetic term, this violates what we know about basic physics.

\footnotetext{
${ }^{12}$ See section $\mathrm{V}$ of $[1$ for an example.

${ }^{13} \mathrm{~A}$ variant is allowing $c$ to vary in the quantity $\rho_{\lambda} \equiv \Lambda c^{2} / 8 \pi G$, see (12)-(15) in [1]; this is equivalent to letting either $\Lambda$ or $G$ vary, as $c$ can be absorbed into the units used to measure $\Lambda$.

${ }^{14}$ This is done for example in [2].
} 
Implication 5: Any varying speed of light theory must be done consistently in terms of its effects on the whole set of physical equations. You can't just allow $c$ to vary in some equations and not in others; and the proposed variation in $c$ in these equations requires some viable physical explanation in order to complete the set of causal relations.

\section{An integrated whole}

The overall message is that you can't just alter the speed of light in one or two equations and leave the rest of physics unchanged. It plays a central role in modern physics 4, particularly because it is the invariant limiting speed of the Lorentz group and so is built into any variables that transform under that group, but also because electromagnetism is central to many physical effects; in particular, light is central to measurement. On the standard view, these various roles are tightly integrated together in a coherent package in which the speed of light does not vary. Any viable VSL theory has to propose a similarly integrated viable alternative to the whole package of physical equations and consequent effects (kinematical and dynamical) dependent on $c$. You can't just tinker with a few bits of the whole.

Acknowledgement: I thank R Durrer and J-P Uzan for helpful comments that have substantially improved this note.

\section{References}

[1] A Albrecht and J Magueijo (1999). "A time varying speed of light as a solution to cosmological puzzles". Phys. Rev. D59 043516 arXiv:astro-ph/9811018.

[2] J D Barrow and J Magueijo (1998). "Varying- $\alpha$ Theories and Solutions to the Cosmological Problems" Phys.Lett. B443 104 astro-ph/9811072.

[3] B A Bassett, S Liberati, C Molina-Paris and M Visser (2000). "Geometrodynamics of variable-speed-of-light cosmologies". Phys Rev D62 10351 arXiv:astro-ph/0001441].

[4] G F R Ellis and J-P Uzan (2005). " 'c' is the speed of light, isn't it?" American Journal of Phyiscs 73, 240 arXiv:gr-qc/0305099.

[5] S W Hawking and G F R Ellis (1973). The Large Scale Structure of Space-Time. (Cmabridge: Cambridge University Press).

[6] G F R Ellis and R M Williams (2000). Flat and Curved Space Times. (Oxford: Oxford University Press).

[7] J.R. Lucas and P.E. Hodgson (1990). Spacetime and Electromagnetism. (Oxford: Clarendon Press).

[8] J Magueijo (2003). "New varying speed of light theories". Rept. Prog. Phys. 662025 arXiv:astro-ph/0305457.

[9] J Moffat (2002). "Variable Speed of Light Cosmology: An Alternative to Inflation" arXiv:hep-th/0208122.

[10] D N Poenaru (2005). "Alexandru Proca, the great physicist". arXiv:physics/0508195.

[11] J L Synge (1960). Relativity: The General Theory (North Holland, Amsterdam).

[12] P Teyssandier (2004). "Variation of the speed of light due to non-minimal coupling between electromagnetism and gravity", Ann. de la Fondation Louis de Broglie, 29, 173 gr-qc/0303081]. 